\title{
A Flexible and Highly Sensitive Piezoresistive Pressure Sensor Based on Micropatterned Films Coated with Carbon Nanotubes
}

\author{
Jia-lin Yao, ${ }^{1,2,3}$ Xing Yang, ${ }^{1,2}$ Na Shao, ${ }^{1,2,4}$ Hui Luo, ${ }^{5}$ Ting Zhang, ${ }^{5}$ and Wu-gui Jiang ${ }^{3}$ \\ ${ }^{1}$ MEMS Laboratory, Department of Precision Instruments, Tsinghua University, Beijing 100084, China \\ ${ }^{2}$ State Key Laboratory of Precision Measurement Technology and Instrumentation, Tsinghua University, Beijing 100084, China \\ ${ }^{3}$ School of Aeronautical Manufacturing Engineering, Nanchang Hangkong University, Nanchang 330063, China \\ ${ }^{4}$ School of Information Engineering, Nanchang Hangkong University, Nanchang 330063, China \\ ${ }^{5}$ i-Lab, Suzhou Institute of Nano-Tech and Nano-Bionics, Chinese Academy of Sciences, Suzhou 215125, China
}

Correspondence should be addressed to Xing Yang; yangxing@tsinghua.edu.cn and Ting Zhang; tzhang2009@sinano.ac.cn

Received 16 April 2016; Accepted 12 July 2016

Academic Editor: Shu Seki

Copyright ( 2016 Jia-lin Yao et al. This is an open access article distributed under the Creative Commons Attribution License, which permits unrestricted use, distribution, and reproduction in any medium, provided the original work is properly cited.

Excellent flexibility, high sensitivity, and low consumption are essential characteristics in flexible microtube pressure sensing occasion, for example, implantable medical devices, industrial pipeline, and microfluidic chip. This paper reports a flexible, highly sensitive, and ultrathin piezoresistive pressure sensor for fluid pressure sensing, whose sensing element is micropatterned films with conductive carbon nanotube layer. The flexible pressure sensor, the thickness of which is $40 \pm 10 \mu \mathrm{m}$, could be economically fabricated by using biocompatible polydimethylsiloxane (PDMS). Experimental results show that the flexible pressure sensor has high sensitivity $\left(0.047 \mathrm{kPa}^{-1}\right.$ in gas sensing and $5.6 \times 10^{-3} \mathrm{kPa}^{-1}$ in liquid sensing $)$ and low consumption $(<180 \mu \mathrm{W})$, and the sensor could be used to measure the pressure in curved microtubes.

\section{Introduction}

Micropressure sensors mainly fabricated by silicon have been used for pressure monitoring and control in many applications, such as altitude sensing [1], flow sensing [2], and level/depth sensing $[2,3]$. However, the demand of sensing the pressure inflexible microtube (e.g., implantable medical devices [4-6], industrial pipeline [7, 8], microfluidic chip $[9,10]$, and smart skin [11]) necessitates prominent features of good flexibility, high sensitivity, and thinner thinness as well as low consumption [12], which may be difficult to meet with silicon sensors.

The micropatterned plastic film method is effective in enhancing sensitivity and ductility and reducing response time of microsensors [13]. For example, Bao's group demonstrated an ultrasensitive resistive pressure sensor with rapid response and a low-pressure regime of $0.8 \mathrm{~Pa}$ by using an elastic microstructured conductive polymer [14]. Wang's group presented a kind of self-powered pressure sensor based on micropatterned plastic films, which have a detection limit of $13 \mathrm{mPa}$ [15]. Cheng's group reported on a new bioinspired strategy to fabricate flexible pressure sensors with touch sensitivity of $50.17 \mathrm{kPa}^{-1}[16]$. Because of the superior mechanical flexibility and great conductivity, carbon nanotube had also been applied as a conductive layer of micropatterned film to further improve the performance of the sensor [13, 17-20]. For instance, Zhang's group presented silk-molded, flexible, micropatterned electronic skin coated with single-walled carbon nanotubes, which has ultrahigh sensitivity $\left(1.8 \mathrm{kPa}^{-1}\right)$ for the detection of minute forces with fast response time [17]. Meanwhile, because of its great conductivity, carbon nanotube applied as a conductive layer may result in low consumption of the flexible sensors [21].

In this paper, a flexible pressure sensor that uses micropatterned films coated with carbon nanotubes is fabricated and is applied to measure the fluid pressure in the curved microtube. Compared with commonly used MEMS pressure sensors, the proposed flexible pressure sensor is thinner and more flexible and has lower consumption. So, the thin and flexible pressure sensor is easier to be installed on the surface of the curved microtube and has less influence on the measured flow field. 


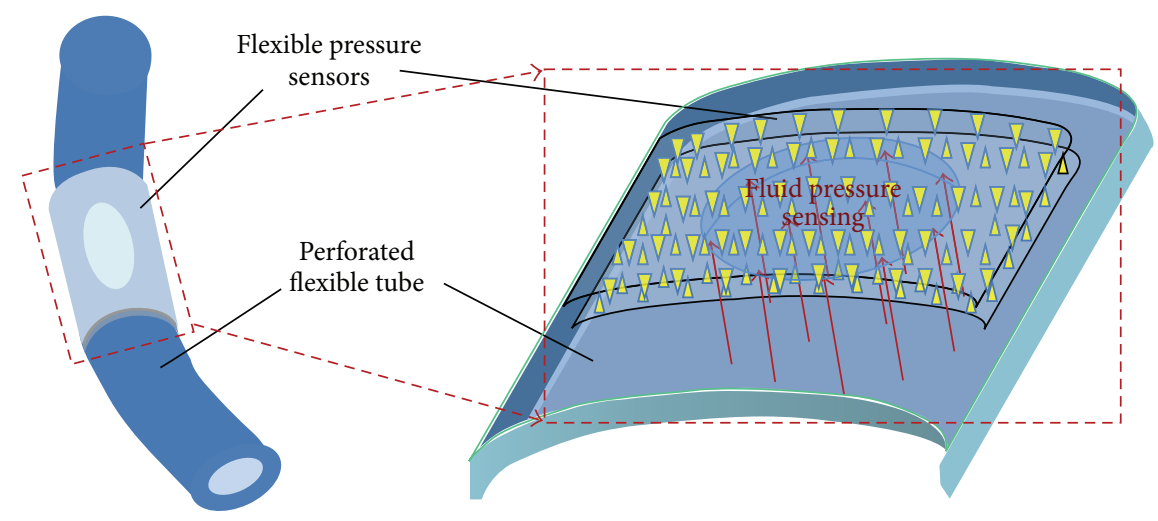

FIGURE 1: Schematic of measuring the flexible microtube's pressure using a flexible pressure sensor.

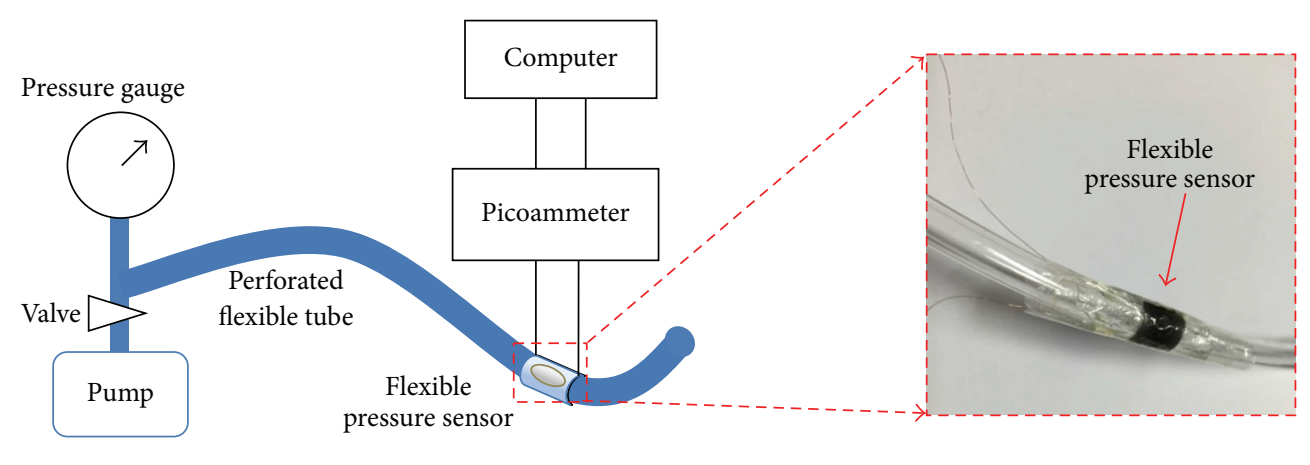

(a)

(b)

FIGURE 2: (a) Diagram of the gas pressure measuring experimental system and (b) photograph of a flexible pressure sensor adhered upon the hole of the perforated flexible microtube.

\section{Fundamentals and Methods}

The pyramidal-shaped micropatterned PDMS layer is applied in our flexible pressure sensor, whose thickness is $40 \pm 10 \mu \mathrm{m}$, and it can be economically fabricated [22]. The schematic of measuring the flexible microtube's pressure using the flexible pressure sensor is shown in Figure 1, which consisted of two PDMS films with micropatterned structure on their contact surfaces for enhancing sensitivity and reducing hysteresis $[14,17,23]$. The PDMS contact surfaces are coated with singlewall carbon nanotube layer to improve conductivity. Given that the resistance of the micropatterned film is very sensitive to the applied vertical force, the flexible pressure sensor is adhered upon the surface of the perforated flexible microtube to sense the vertical force which is generated by the fluid pressure in the microtube. Thus, the wall pressure of the microtube could be measured in real time by monitoring the resistance of the micropatterned film. Two examples for measuring gas and liquid pressure were provided as follows.

According to the fundamentals and analysis mentioned above, the gas pressure measuring experimental system is illustrated in Figure 2(a), which comprises a flexible pressure sensor, a perforated flexible microtube, a pump, a valve, a standard pressure gauge, a picoammeter, and a computer. Herein, the flexible pressure sensor is adhered upon the hole on the perforated flexible microtube's surface. The gas

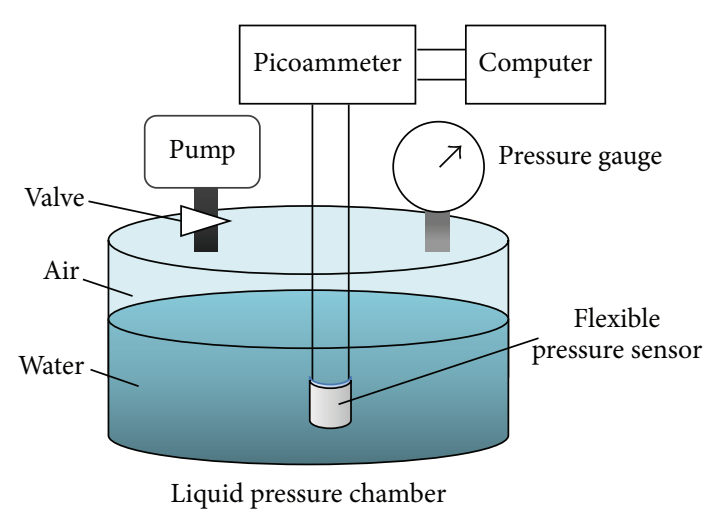

FIGURE 3: Diagram of the liquid pressure experimental system.

pressure in the flexible microtube is monitored by the standard pressure gauge and is adjusted by the pump as well as the valve. The electric output signal of the flexible pressure sensor is recorded in real time by the picoammeter. The pressure in the tube will be measured when the micropatterned film is subjected to pressure through the hole on the tube, as shown in Figure 2(b). The computer receives the measurement data and then processes and displays the results. 


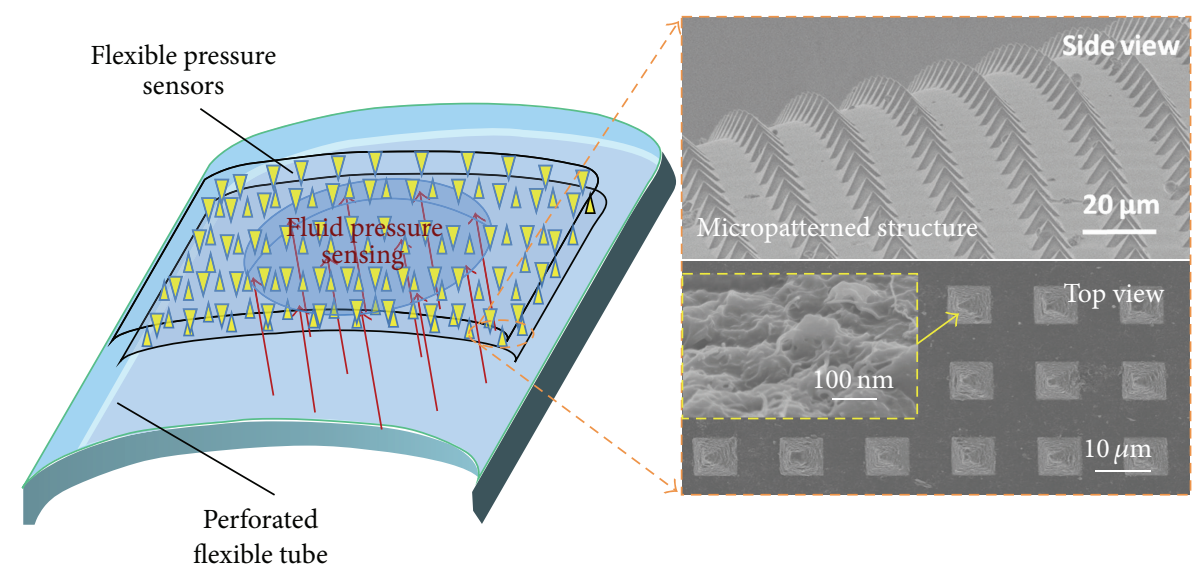

FIGURE 4: SEM images of the micropatterned film.

Figure 3 shows another method that utilizes the flexible sensor to measure the liquid pressure. In this case, the flexible pressure sensor is directly attached to the curved surface of the sealed liquid pressure chamber. The gas pressure transfers to the liquid, and then the liquid pressure is transmitted to the flexible pressure sensor attached to the wall of the liquid chamber. The liquid pressure in the chamber is also measured by the standard pressure gauge and is adjusted by the pump as well as the valve. Other parts of the experimental system are similar to those in the gas experimental system: the electric output signal of the flexible pressure sensor is recorded in real time by the picoammeter. The computer receives the measurement data and then processes and displays the results.

\section{Results and Discussion}

Figure 4 shows the SEM images of the pyramidal-shaped micropatterned film, which is an array of pyramid microstructures made by PDMS coated with conductive single-wall carbon nanotube layer (inset in the top view SEM image). The experimental results of measuring the gas pressure are shown in Figure 5. There is a positive correlation relationship between gas pressure and current. The sensor's sensitivity $S$ can be defined as

$$
\begin{aligned}
S & =\frac{\delta\left(\Delta I / I_{0}\right)}{\delta p}, \\
\Delta I & =I-I_{0},
\end{aligned}
$$

where $I$ is the pressure sensor's current when pressure is applied on the device; $I_{0}$ is the pressure sensor's current in the condition of the initial pressure; $p$ is the applied pressure [17].

As it is shown in Figure 5, the curves of the current versus the pressure could be divided into two appreciated linear parts. In the low-pressure region $(0-30 \mathrm{mmHg})$, the sensor has higher sensitivity $\left(4.7 \times 10^{-2} \mathrm{kPa}^{-1}\right)$ calculated by (1), while, in the range from 4 to $13.3 \mathrm{kPa}(30-100 \mathrm{mmHg})$, the sensitivity of the sensor is $1.1 \times 10^{-3} \mathrm{kPa}^{-1}$. And the

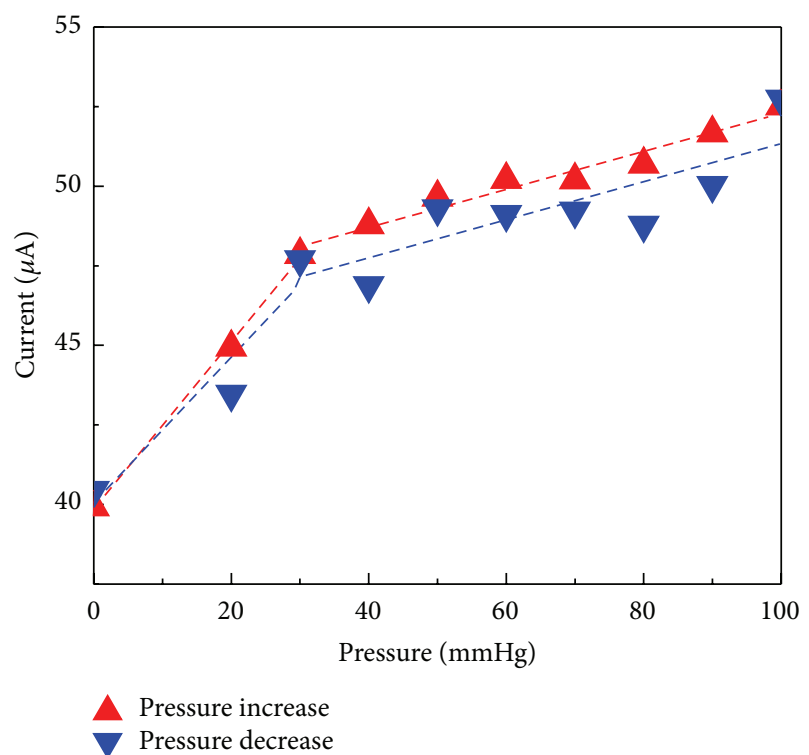

FIgURE 5: Gas pressure in the curved tube versus current at constant voltage $=1 \mathrm{~V}$. The red curve is the output current with the increase of pressure; the blue curve is the output current with the decrease of pressure.

curves of liquid pressure versus current of the sensor are shown in Figure 6, which are also positively related. The calculations show that the proposed sensor also has high sensitivity $\left(5.6 \times 10^{-3} \mathrm{kPa}^{-1}\right)$ in the low-pressure region $(0-$ $140 \mathrm{mmHg}$ ). The sensitivity of the sensor is $1.8 \times 10^{-3} \mathrm{kPa}^{-1}$ when the liquid pressure changes from 140 to $300 \mathrm{mmHg}$. The flexible sensors show lower sensitivity in the high-pressure region, which is mainly determined by the characteristics of pyramidal-shaped microstructures. When the pressure comes to the high region, the deformation of the peak of pyramidal-shaped microstructures is harder than the lowpressure region, which results in lower sensitivity. In both cases, the proposed sensors all have low power consumption $(<55 \mu \mathrm{W}$ and $<180 \mu \mathrm{W}$, resp.). 


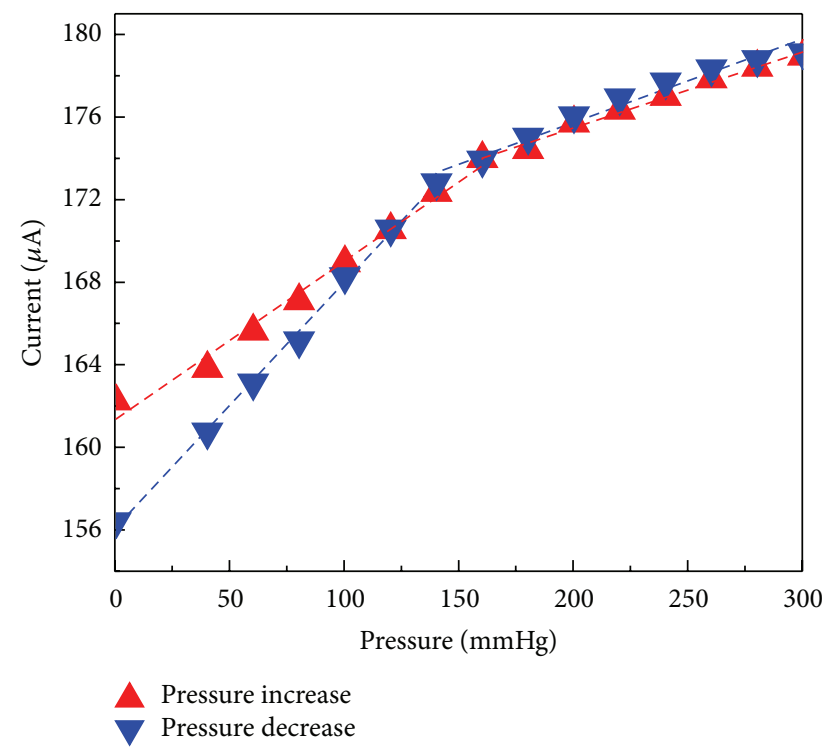

FIGURE 6: Liquid pressure versus current at constant voltage $=1 \mathrm{~V}$. The red curve is the output current with the increase of pressure; the blue curve is the output current with the decrease of pressure.

The experimental results show that, compared with the commonly used MEMS pressure sensors, the proposed flexible pressure sensors have higher sensitivity, lower consumption, and ultrathin size. For instance, NovaSensor's NPC-100, a type of commercial MEMS pressure sensor, has pressure sensitivity of $3.75 \times 10^{-5} \mathrm{kPa}^{-1}$ and consumption of $20 \mathrm{~mW}$ with a thickness of $4.32 \mathrm{~mm}$ [24]. Moreover, the advantages of the sensor also include that the sensor's measurement range could be adjusted by selecting different parameters of the micropatterned film. Furthermore, due to its high flexibility and ductility, the sensor could be easily attached to the curve surface of the microtube with minute impact on the flow field.

\section{Conclusion}

In this paper, we demonstrate a flexible, highly sensitive, low consumption, and ultrathin sensor based on pyramidalshaped micropatterned films coated with carbon nanotubes for measuring the fluid pressure in the curved microtube. The experimental results show that the flexible sensors can conveniently measure the pressure of the liquid and the gas in the curved microtube. Moreover, the flexible sensor has high pressure sensitivity of $4.7 \times 10^{-2} \mathrm{kPa}^{-1}$ in gas sensing, 5.6 $\times 10^{-3} \mathrm{kPa}^{-1}$ in liquid sensing, low consumption $(<180 \mu \mathrm{W})$, and ultrathin thickness of $40 \pm 10 \mu \mathrm{m}$. We envision that the flexible pressure sensors could be used in implantable medical pressure sensing, industrial pipeline pressure sensing, microfluidic chip pressure sensing, and smart skin pressure sensing.

\section{Competing Interests}

The authors declare that they have no competing interests.

\section{Acknowledgments}

This work was supported by the National Natural Science Foundation of China (Grants nos. 51375263, 11274190, and 61574163) and the National Key Basic Research Program of China (no. 2013CB934200).

\section{References}

[1] J. S. Jang and D. Liccardo, "Automation of small UAVs using a low cost MEMS sensor and embedded computing platform," in Proceedings of the IEEE 25th Digital Avionics Systems Conference, pp. 1-9, Portland, Ore, USA, October 2006.

[2] A. G. P. Kottapalli, M. Asadnia, J. M. Miao, C. W. Tan, G. Barbastathis, and M. Triantafyllou, "Polymer MEMS pressure sensor arrays for fish-like underwater sensing applications," Micro \& Nano Letters, vol. 7, no. 12, pp. 1189-1192, 2012.

[3] A. V. Chavan and K. D. Wise, "Batch-processed vacuum-sealed capacitive pressure sensors," Journal of Microelectromechanical Systems, vol. 10, no. 4, pp. 580-588, 2001.

[4] S. Gong, W. Schwalb, Y. Wang et al., "A wearable and highly sensitive pressure sensor with ultrathin gold nanowires," Nature Communications, vol. 5, article 3132, 2014.

[5] P.-J. Chen, D. C. Rodger, S. Saati, M. S. Humayun, and Y.-C. Tai, "Microfabricated implantable parylene-based wireless passive intraocular pressure sensors," Journal of Microelectromechanical Systems, vol. 17, no. 6, pp. 1342-1351, 2008.

[6] C. Li, P.-M. Wu, L. A. Shutter, and R. K. Narayan, "Dualmode operation of flexible piezoelectric polymer diaphragm for intracranial pressure measurement," Applied Physics Letters, vol. 96, no. 5, Article ID 053502, 2010.

[7] M. D. Kalman, L. Yu, D. Moosberg, and D. Johnson, "Development and testing of a non-interlocked hoop strength layer for unbonded flexible pipe," in Proceedings of the Offshore Technology Conference, Houston, Tex, USA, 2011.

[8] G. T. Ashcombe and R. C. Kenison, "The problems associated with NDT of high pressure flexible pipes," in Advances in Subsea Pipeline Engineering and Technology, vol. 24 of Advances in Underwater Technology, Ocean Science and Offshore Engineering, pp. 179-204, Springer, Dordrecht, The Netherlands, 1990.

[9] H. Jin, J. Zhou, X. He et al., "Flexible surface acoustic wave resonators built on disposable plastic film for electronics and lab-on-a-chip applications," Scientific Reports, vol. 3, article 2140, 2013.

[10] S. Xu, Y. Zhang, L. Jia et al., "Soft microfluidic assemblies of sensors, circuits, and radios for the skin," Science, vol. 344, no. 6179, pp. 70-74, 2014.

[11] T. V. Papakostas, J. Lima, and M. Lowe, "A large area force sensor for smart skin applications," IEEE Sensors Journal, vol. 2, pp. 1620-1624, 2002.

[12] C. Pang, C. Lee, and K.-Y. Suh, "Recent advances in flexible sensors for wearable and implantable devices," Journal of Applied Polymer Science, vol. 130, no. 3, pp. 1429-1441, 2013.

[13] G. Schwartz, B. C.-K. Tee, J. Mei et al., "Flexible polymer transistors with high pressure sensitivity for application in electronic skin and health monitoring," Nature Communications, vol. 4, article 1859, 2013.

[14] L. Pan, A. Chortos, G. Yu et al., "An ultra-sensitive resistive pressure sensor based on hollow-sphere microstructure induced elasticity in conducting polymer film," Nature Communications, vol. 5, article 3002, 2014. 
[15] F.-R. Fan, L. Lin, G. Zhu, W. Wu, R. Zhang, and Z. L. Wang, "Transparent triboelectric nanogenerators and self-powered pressure sensors based on micropatterned plastic films," Nano Letters, vol. 12, no. 6, pp. 3109-3114, 2012.

[16] B. Su, S. Gong, Z. Ma, L. W. Yap, and W. Cheng, "Mimosainspired design of a flexible pressure sensor with touch sensitivity," Small, vol. 11, no. 16, pp. 1886-1891, 2015.

[17] X. Wang, Y. Gu, Z. Xiong, Z. Cui, and T. Zhang, "Silk-molded flexible, ultrasensitive, and highly stable electronic skin for monitoring human physiological signals," Advanced Materials, vol. 26, no. 9, pp. 1336-1342, 2014.

[18] C. Guo, Y. Yu, and J. Liu, "Rapidly patterning conductive components on skin substrates as physiological testing devices via liquid metal spraying and pre-designed mask," Journal of Materials Chemistry B, vol. 2, no. 35, pp. 5739-5745, 2014.

[19] J. Reeder, M. Kaltenbrunner, T. Ware et al., "Mechanically adaptive organic transistors for implantable electronics," Advanced Materials, vol. 26, no. 29, pp. 4967-4973, 2014.

[20] T. Sekitani and T. Someya, "Stretchable organic integrated circuits for large-area electronic skin surfaces," MRS Bulletin, vol. 37, no. 3, pp. 236-245, 2012.

[21] W.-D. Zhang and W.-H. Zhang, "Carbon nanotubes as active components for gas sensors," Journal of Sensors, vol. 2009, Article ID 160698, 16 pages, 2009.

[22] S. C. B. Mannsfeld, B. C.-K. Tee, R. M. Stoltenberg et al., "Highly sensitive flexible pressure sensors with microstructured rubber dielectric layers," Nature Materials, vol. 9, no. 10, pp. 859-864, 2010.

[23] S. Park, H. Kim, M. Vosgueritchian et al., "Stretchable energyharvesting tactile electronic skin capable of differentiating multiple mechanical stimuli modes," Advanced Materials, vol. 26, no. 43, pp. 7324-7332, 2014.

[24] G. E. Sensing, "NPC-100 Series NovaSensor Disposable Medical Pressure Sensors," 2006. 

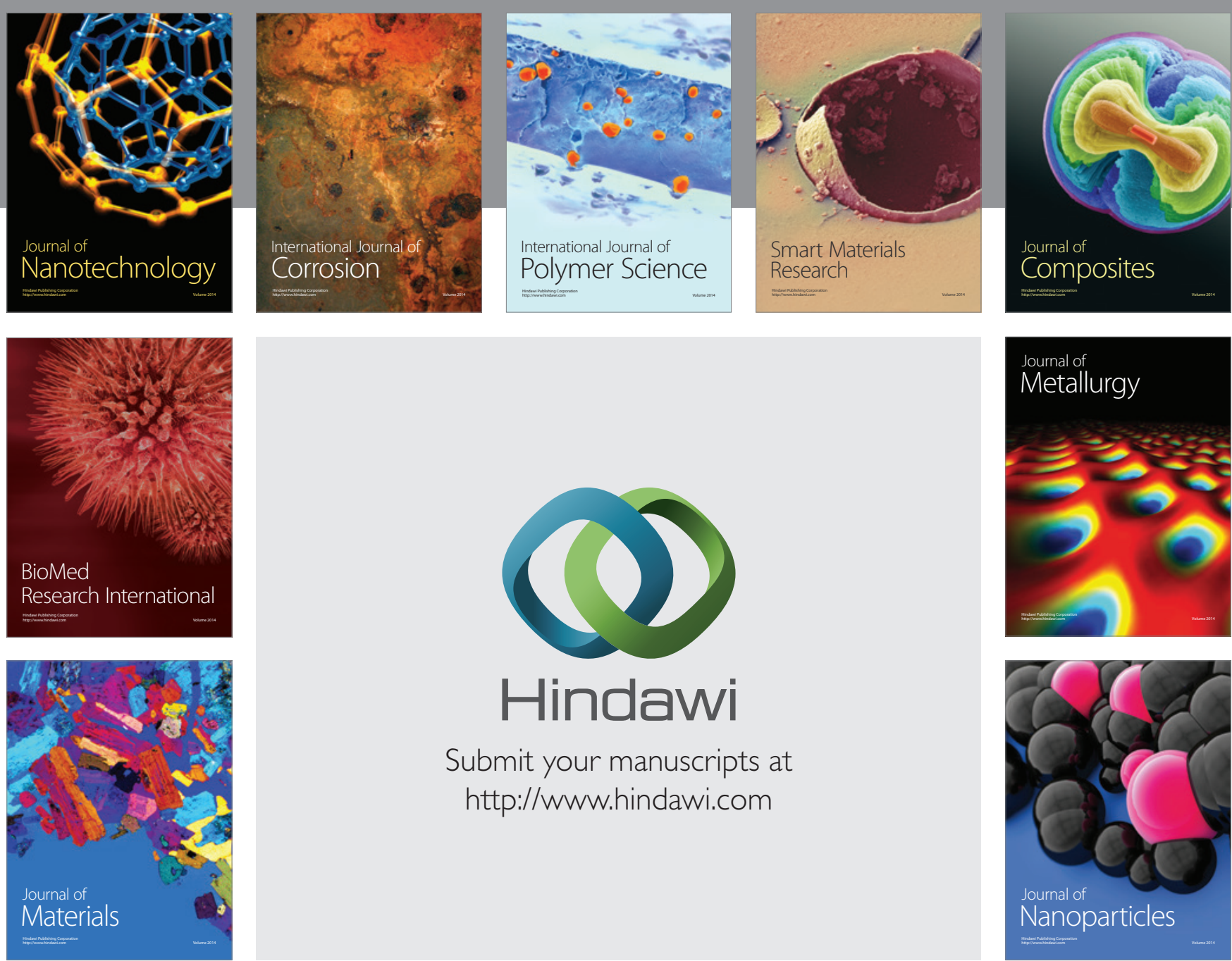

\section{Hindawi}

Submit your manuscripts at

http://www.hindawi.com

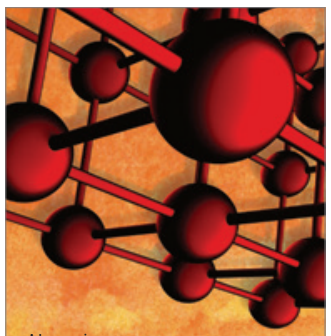

Materials Science and Engineering
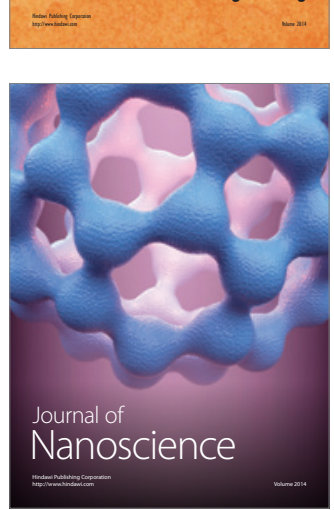
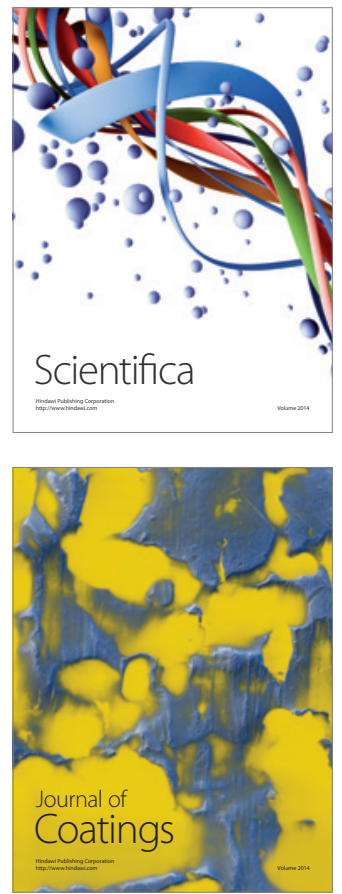
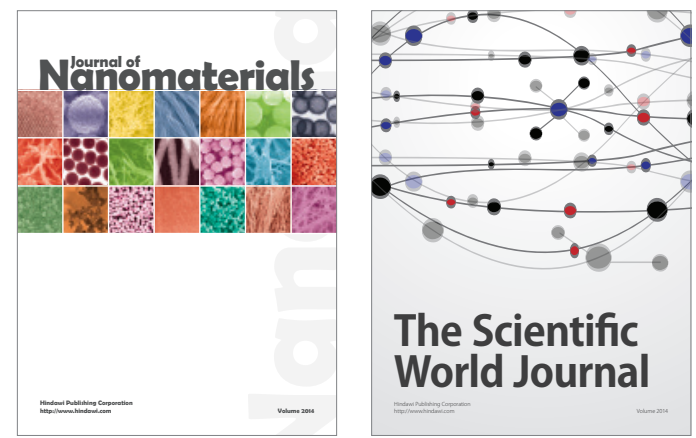

The Scientific World Journal
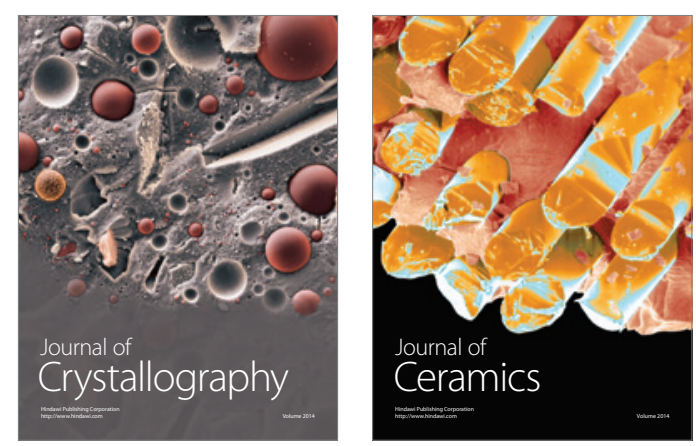
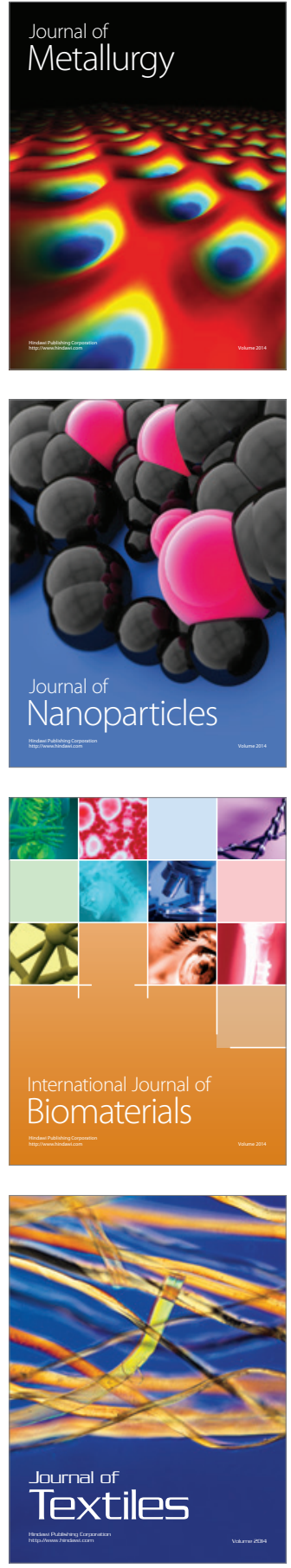